\title{
Investigating Cognitive Performance Deficits in Male and Female Soccer Players after a 4-week Heading- Training Programme: A Controlled Study
}

\author{
Petra Jansen and Jennifer Lehmann \\ Institute of Sport Science, University of Regensburg, Regensburg, Germany
}

\begin{abstract}
This experimental study with a controlled pre-test and post-test group design investigated the possible effects of four sessions of heading training on cognitive performance. A total of 118 participants (mean age of 22.25 years) were assigned to one of the three following groups: a heading-training group, a passingtraining group and a waiting control group. The participants completed a cognitive test battery of attention (D2-test), working memory (Paced Auditory Serial Addition Task, PASAT-2) and visual spatial intelligence (Mental Rotation Test) tests before, immediately after the second training session and one week after the fourth training session. There were no between-group differences in cognitive performance after the second or the fourth heading training sessions. However, within the heading-training group, women complained more of headaches than men after the fourth training session. These results build on the results from the study of Rieder and Jansen (2011) that showed no neuropsychological consequences after one heading session, showing no cumulative effects of repeat heading.
\end{abstract}

Keywords: soccer, heading, cognitive impairment, sex effect, headache, concussion, mild traumatic brain injury

Soccer is very popular with more than 265 million players around the world (see Stephens, Rutherford, Potter, \& Fernie, 2010). Even though millions of players are fascinated with this sport, it bears a high risk of injuries, especially in the lower extremities (knees and ankles). In addition, head injuries also play an important role (Dvorak \& Junge, 2000). The head injuries can be differentiated into concussions arising due to (a) collisions or (b) the impact of headings, with the latter further differentiated into cumulative and acute events. Concussions can be subsumed under the term of 'traumatic brain injury' (TBI). Symptoms include headaches, memory loss and vegetative symptoms such as dizziness or nausea. More specifically, Rutherford and Stephens (2007) characterized mild TBI as including neck ache, vegetative symptoms and brief changes of consciousness. Since November 2015, much attention has been focused on the possible negative impacts of heading in the media around the world. This was due to the United States Soccer Federation banning players less than 11 years of age from heading. Furthermore, they announced that for 11-13-years-old's, headers should be reduced. Although it is well known that concussions due to collisions lead to neurological complaints (Talavage et al., 2014), the neurological impact of heading is not well investigated. 


\section{Effects of Cumulative and Acute Heading}

\section{Cumulative Effects}

When investigating cumulative effects, elite athletes have often been compared to non-elite athletes and their heading activity has been estimated retrospectively. Therefore, those cumulative effects were mostly investigated in quasiexperimental designs (e.g., Stephens et al., 2010). The results showed, for example, that elite soccerplayers suffered from brain impairments which were comparable to those of patients with brain lesions. This effect was not visible in brain-imaging studies (Koerte, Ertl-Wagner, Reiser, Zafonte, $\&$ Shenton, 2012). Furthermore, soccer players showed a cortical thinning in the inferolateralparietal, temporal and occipital cortex, which was associated with lower cognitive performance (Koerte et al., 2016). Lipton et al. (2013) conducted a study with diffusion-tensor magnetic resonance imaging to quantify the impact of heading in soccer players, with the impact of heading quantified retrospectively. It was shown that extensive heading-training related to microstructural differences in white matter and a decline in memory performance (Lipton et al., 2013). In a review by Mahler, Hutchinson, Cusimano, Comper and Schweizer (2014), it is concluded that the neurocognitive outcome of concussions in soccer is similar to that found with other sports. Otherwise stated, with the studies at hand, one cannot conclude that concussions in soccer lead to different effects than concussions with other types of sport. This shortcoming is related to the fact that studies were mostly retrospective. Furthermore, it should be discussed if brain changes following prolonged soccer training must be perceived as brain damage or rather represent an adaptive reaction of the brain.

\section{Acute Effects}

Concerning acute effects of heading on cognitive performance, there are, to our knowledge, only four studies. Putukian, Echemendia and Mackin (2000) investigated the impact of heading-training compared to passing-training in a group of adults. The authors did not find any change in cognitive functions due to the heading-training. However, it might be possible that in this study, the practice effects counteracted acute changes in cognitive function. Every participant served as his own control and underwent the testing three times. A control group was missing.

This methodological flaw was overcome by a study of Rieder and Jansen (2011). In this experimental study, a pre-test and post-test de- sign with three groups (one heading group, one passing group and one waiting control group), who were matched according to their age, sex and IQ were used. Instantly after the training, neuropsychological tests of attention and working memory were conducted. The results showed that the heading-training did not affect the neuropsychological performance. However, within the heading group, women complained more about headaches than men. The no-effect result was confirmed by a study of Dorminy et al. (2015). They showed in a controlled setting that an acute bout of soccer heading across various ball velocities neither affected S-100B serum concentration (ng $\mathrm{mL}^{-1}$ ) nor the performance in concussion test scores. In a recently published study, the authors (Elbin et al., 2015) investigated a heading-training of 15 minutes with and without protective soccer headgear. Before and after the heading-training, neurocognitive performance and symptom reports were assessed. Participants wearing soccer headgear showed worse performance concerning verbal memory than the group without headgear. Moreover, this control group, the group without headgear, improved their reaction time compared to the headgear-group. Thus, in this study, head protection did not protect from possible cognitive decline due to heading.

\section{The Role of Gender Concerning the Impact of Heading}

Gender differences in soccer are well known including different kinematics such as a higher joint velocity for the knee and the hip while kicking for males (Katis, Kellis, \& Lees, 2015), or a sudden stiffening of the knee and the lower leg while landing in a single-leg task (Lephart, Ferris, Riemann, Myers, \& Fu, 2002). Concerning concussion, women seem to suffer more than men (Covassin \& Elbin, 2011). It was shown that women have a higher risk of sustaining a concussion, and need a longer time to recover (Covassin, Swanik, $\&$ Sachs, 2003). Differences between men and women in concussion recovery have also been investigated by Colvin et al. (2009). Their results showed that female soccer players had a lower performance than males on computer-based neuropsychological testing (reaction time, memory, visual motor-speed composite scores) with the ImPACT test battery. Furthermore, women complained more about concussion symptoms.

\section{Goal of the Present Study}

The present study builds on the earlier study of Rieder and Jansen (2011) by extending the time of 
training from one session to four sessions with 100 headings each. According to the study of Lipton et al. (2013), the median for one-year retrospective heading experience was 432 headings per year. For this, the influence of heading in four sessions of 100 headings each seemed to be reasonable to investigate in more detail, using a prospective design. It was hypothesized that a total of 400 headings in four sessions is more likely to lead to cognitive impairments than 100 headings conducted in one session. Furthermore, based on the results from Rieder and Jansen (2011), it was hypothesized that women would complain more about symptoms in the heading group than men.

\section{Methods}

\section{Participants}

The selected sample for this study included 118 healthy students of sport science at a University (69 females, 49 male). Sports students were chosen as participants due to the following reason: first to achieve comparability to the study of Rieder and Jansen (2011) and second, it could be assumed that they know how to play soccer and could perform headings correctly. The average age of the sample was 22.25 years $(S D=2.39)$, with a mean age of $23.02(S D=2.77)$ years for the male participants and $21.70(S D=1.92)$ years for the female participants. Three groups were investigated in this study: a heading-training group (TG), a passingtraining control group (PCG) and a waiting control group (WCG). Each of the groups consisted of male and female subgroups: TG (19 males, 16 females), PCG (21 males, 18 females) and the WCG (9 males, 35 females). All the participants had already played soccer in some way before, but the two active groups did not differ regarding the intensity of their former soccer training (measured as training time per week; Kruskal-Wallis, $p=.79$ ) and the level of the league (between the 9th and 4th highest class in the German-soccer classification) (Kruskal-Wallis, $p=.85$ ). All participating students gave their voluntary informed consent.

\section{Materials}

The test battery was similar to the one used in the study by Rieder and Jansen (2011) and was chosen to achieve comparability. It comprised a PersonalData Questionnaire (see Rieder \& Jansen, 2011), a Cognitive Speed Measurement (ZVT; Oswald \& Roth, 1987), a Neuropsychological Test Battery measuring attention, working memory, and speed of information processing (D2-test, Brickenkamp, 2002; Paced Auditory Serial Addition Task, PASAT, Gronwall, 1977), a Mental Rota- tion Test (MRT; Vandenberg \& Kuse, 1978) and a Physical Questionnaire.

The ZVT is the equivalent of the Trail Making Test A (Reitan, 1956). In this test, participants are asked to connect the numbers on a sheet of paper in ascending order as fast as possible within the time limit of 30 seconds. The internal consistency as well as 6 months of test-retest reliability of the ZVT is about .90 to .95 . The D2-Test is a letter cancellation test that taps concentration and selective attention. The dependent variable is the "Concentration-Performance Score (CPS)'. The CPS correlates with a value of .78 with the mosaic-test of the 'Hamburg-WechslerIntelligence Test for Adults - HAWIE'. The testretest reliability for 4-18 days is .93.

The speed of information processing, sustained attention and working memory were assessed with the Paced Auditory Serial Addition Task (PASAT). The test-retest coefficients across the testing intervals spanning $0,5,6,12$ and 18 months range from .9 to .96 (McCaffrey, Westervelt, \& Haase, 2001). The MRT is a paper-pencil test in which the spatial ability is assessed to mentally imagine what an object would look like when rotated. Due to the different test times in this study and to exclude a learning effect, versions A, B and C of the MRT were used.

The tests measuring working memory as well as attention were chosen because the cognitive performances are processed at least partly in the prefrontal cortex, which in turn is the brain's region most affected by heading (compare to Witol \& Webbe, 2003). Because concussion symptoms are only expected only for the heading group (see Rieder \& Jansen, 2011), the Physical Questionnaire, which was constructed for this study, was administered only to this group. The questionnaire comprised four items: (a) Do you have a headache now? (b) Did you have a headache during the training? (c) Do you feel nauseated? (d) How intensive was the headache? The first three questions had to be answered with 'yes $=1$ ' or 'no $=0$ '; the fourth question with a number between 1 (no complaints) and 10 (strong complaints).

\section{Training Intervention}

The heading-training in the TG was based on the guidelines of the German Soccer Association (Deutscher Fußballbund) and included four different exercises. Before each of the training session, a warm-up was performed and each of the relevant techniques was presented to the participants (e.g., heading while standing, heading while jumping). In the heading group, around 100 headings were conducted per session. Thereby, 
the heading tasks were varied (i.e., heading had to be conducted from different positions in the field, with different partners, etc.). The participants in the PCG received passing-training without any heading-training at all. The training lasted for 4 weeks with one-hour training sessions per week.

\section{Procedure}

The study comprised three different test times. All groups were tested at the beginning, immediately after the second and one week after the fourth training session. At the first testing point (Pre-test), all participants completed the Personal-Data Questionnaire and afterwards completed the ZVT, MRTA, D2 and the PASAT with the three- and twosecond variation. The ZVT was only used at Pretest because it served as a control-measurement. The Post-test immediately after the second session (Post-2) and one week after the four-week intervention (Post-4) for all groups, consisted of the MRT-B (after second session) and MRT-C (after fourth session), D2 and the PASAT. The tests were administered in the order they are mentioned here. The duration of each testing session was around 50 minutes. We decided to conduct the Post- 4 test one week after the fourth session to test the immediate effect of the heading-training. Only the participants of the TG had to answer the Physical Questionnaire at the Post-4 testing point.

\section{Statistical Methods}

To analyse the neuropsychological data, $3 * 2 * 3$ analyses of variance with test time (Pre-test, Post2 session and Post-4 session) as a within-subject factor and group (TG, PCG, WCG) and sex (male, female) as between subject factors for each of the dependent variables (D2-Test, PASAT-Test, MRT) were conducted. Because the participants of each group differed in their cognitive processing speed, $\left(F(2,115)=3.34, p<.05, \eta^{2}=.06\right)$, the ZVT was used as a co-variate. For the analysis of the items of the Physical Questionnaire, chi-square and Mann Whitney U-Tests were used. This was applied to examine differences between the groups concerning the frequency of headache during and after the training, and nausea after the training. The significance level of the test was corrected according to the Bonferroni-method, resulting in a significance level of $\alpha=.005$.

\section{Results}

All descriptive data of the three cognitive tests are shown in Table 1.

\section{MRT}

The only effects we found were significantly better performance of males compared to females, $\left(F(1,95)=23.81, p<.001, \eta^{2}=.20\right)$. This result was qualified $\left(F(2,95)=4.80, p<.05, \eta^{2}=.09\right)$ by the fact that the mental rotation performance of the males and females did not differ in the TG, (TG: $F(1,32)=.95$, n.s.), but in both of the other groups (PCG: $F(1,36)=11.31, p<.01, \eta^{2}=.24$; WCG: $\left.F(1,43)=21.93, p<.001, \eta^{2}=.35\right)$. In these two groups, males showed better performance than females.

\section{D2}

The analysis of variance showed at first that the performance was better after the Post- 2 session and Post -4 session compared to the Pre-test $(F(2$, $\left.170)=5.885, p<.005, \eta^{2}=.07\right)$. Furthermore, it was shown that there was no performance difference in the TG and PCG between males and females, but in the WCG, females showed better performance than males $(F(2,170)=3.78, p<.05$, $\left.\eta^{2}=.04\right)$. A significant three-way interaction between group, sex and test time, $(F(4,170)=3.95$, $p<.01, \eta^{2}=.09$ ) showed that the effects mentioned above should be analysed in detail. The relevant result here is that the difference between the Pre-test and both the Post- 2 session and Post- 4 session was larger in the PCG for females compared to males $\left(F(1,25)=13.40, p<.001, \eta^{2}=.35\right)$.

\section{PASAT}

The only two effects occurring in the 2-sec PASAT were that (i) males showed a better performance than females $\left(F(1,94)=11.07, p<.01, \eta^{2}=.11\right)$ and that (ii) the performance was better in the Post2 session and Post- 4 session compared to the pretest $\left(F(2,188)=73.20, p<.05, \eta^{2}=.03\right)$. The only effect in the 3-sec PASAT was that males showed better performance than females $(F(1,95)=5.027$, $\left.p<.05, \eta^{2}=.05\right)$.

\section{Physical Questionnaire}

The number of males and females who suffered from symptoms is given in Table 2. Females reported the presence of headaches more frequently than males during the training $\left.\chi^{2}(1, N=33)=5.66, p<.05\right)$. They also complained more frequently about headaches than males shortly after the training $\chi^{2}(1$, $N=33)=8.56, p<.01)$. Furthermore, the intensity of the headaches was higher for females than for males $(U(18,15)=69.5, p<.05)$. 
TABLE 1

Means and Standard Deviations Dependent on Time of Testing and Group for the Four Cognitive Tests, MRT, D2, PASAT 2 and 3

\begin{tabular}{|c|c|c|c|c|c|c|c|c|c|c|c|c|}
\hline & \multicolumn{3}{|c|}{ MRT (correct items) } & \multicolumn{3}{|c|}{ D2 (CPS) } & \multicolumn{3}{|c|}{ PASAT 2 (percent correct) } & \multicolumn{3}{|c|}{ PASAT 3 (percent correct) } \\
\hline & Pre-test & $\begin{array}{l}\text { Post-2 } \\
\text { session }\end{array}$ & $\begin{array}{l}\text { Post-4 } \\
\text { session }\end{array}$ & Pre-test & $\begin{array}{l}\text { Post-2 } \\
\text { session }\end{array}$ & $\begin{array}{l}\text { Post-4 } \\
\text { session }\end{array}$ & Pre-test & $\begin{array}{l}\text { Post-2 } \\
\text { session }\end{array}$ & $\begin{array}{l}\text { Post-4 } \\
\text { session }\end{array}$ & Pre-test & $\begin{array}{l}\text { Post-2 } \\
\text { session }\end{array}$ & $\begin{array}{l}\text { Post-4 } \\
\text { session }\end{array}$ \\
\hline TG & $\begin{array}{l}13.26 \\
(4.28)\end{array}$ & $\begin{array}{l}17,21 \\
(4.57)\end{array}$ & $\begin{array}{l}10.06 \\
(4.03)\end{array}$ & $\begin{array}{l}208.53 \\
(39.05)\end{array}$ & $\begin{array}{l}246.53 \\
(38.40)\end{array}$ & $\begin{array}{l}253.40 \\
(38.18)\end{array}$ & $\begin{array}{l}63.32 \\
(23.68)\end{array}$ & $\begin{array}{l}78.17 \\
(17.90)\end{array}$ & $\begin{array}{l}81.56 \\
(16.77)\end{array}$ & $\begin{array}{l}91.27 \\
(10.74)\end{array}$ & $\begin{array}{l}97.29 \\
(4.24)\end{array}$ & $\begin{array}{l}98.08 \\
(3.23)\end{array}$ \\
\hline PCG & $\begin{array}{l}12.07 \\
(4.75)\end{array}$ & $\begin{array}{l}16.76 \\
(5.37)\end{array}$ & $\begin{array}{l}8.69 \\
(4.00)\end{array}$ & $\begin{array}{l}204.61 \\
(37.47)\end{array}$ & $\begin{array}{l}235.10 \\
(40.37)\end{array}$ & $\begin{array}{l}242.90 \\
(41.05)\end{array}$ & $\begin{array}{l}73.19 \\
(22.41)\end{array}$ & $\begin{array}{l}85.69 \\
(14.21)\end{array}$ & $\begin{array}{l}83.33 \\
(17.36)\end{array}$ & $\begin{array}{l}91.57 \\
(11.76)\end{array}$ & $\begin{array}{l}98.16 \\
(16.41)\end{array}$ & $\begin{array}{l}98.08 \\
(3.51)\end{array}$ \\
\hline WCG & $\begin{array}{l}11.13 \\
(4.69)\end{array}$ & $\begin{array}{l}14.51 \\
(5.55)\end{array}$ & $\begin{array}{l}8.02 \\
(4.32)\end{array}$ & $\begin{array}{l}213.97 \\
(35.09)\end{array}$ & $\begin{array}{l}247.36 \\
(32.86)\end{array}$ & $\begin{array}{l}258.07 \\
(30.32)\end{array}$ & $\begin{array}{l}66.87 \\
(21.52)\end{array}$ & $\begin{array}{l}83.52 \\
(13.39)\end{array}$ & $\begin{array}{l}85.12 \\
(12.99)\end{array}$ & $\begin{array}{l}94.39 \\
(7.02)\end{array}$ & $\begin{array}{l}97.43 \\
(4.80)\end{array}$ & $\begin{array}{l}98.00 \\
(2.30)\end{array}$ \\
\hline
\end{tabular}




\section{TABLE 2}

Number (Percent) of Males and Females Who Complained of Neuropsychological Symptoms

\begin{tabular}{lcccl}
\hline & $\begin{array}{l}\text { Headache during } \\
\text { the training }\end{array}$ & $\begin{array}{l}\text { Headache after } \\
\text { the training }\end{array}$ & $\begin{array}{l}\text { Intensity of } \\
\text { headache }\end{array}$ & $\begin{array}{l}\text { Nausea during the } \\
\text { training }\end{array}$ \\
\hline Males (18) & $7(39 \%)$ & $3(17 \%)$ & $3(17 \%)$ & $0(0 \%)$ \\
Females (15) & $12(80 \%)$ & $10(67 \%)$ & $7(47 \%)$ & $0(0 \%)$ \\
\hline
\end{tabular}

Note. Given are the numbers of people who scored the intensity of headache as moderate, strong and very strong $1 \geq 4$ on a scale from 0 to 10$)$.

\section{Discussion}

The results show that there were no negative consequences of 400 headings on cognitive functions. Furthermore, and as hypothesized, women complained more about symptoms in the heading group than males. The results of the current prospective study are in line with the study of Rieder and Jansen (2011). The study extended the former work by showing no effects after four sessions of heading-training, which was based on the normal practice routines in soccer clubs. The cognitive tasks investigated in this study included attention, working memory as well as visual spatial intelligence. Those tasks are processed at least partly in the prefrontal cortex, the brain region affected by heading. Thus, we conclude that the tests would be sensitive to detecting cognitive impairment due to heading. Furthermore, the PASAT test was developed to differentiate between persons who had sustained a concussion and uninjured persons. In summary, it seems that even if the training was extended from one (Rieder \& Jansen, 2011) to four sessions, cognitive performance did not decline.

\section{The Importance of the Amount of Heading}

At first glance, this seems to contradict retrospective studies that heading-training leads to changes in the brain, both at a neurocognitive level (e.g., visual working memory deficits), as well as neurophysiological level (e.g., altered activation in the dorsolateral prefrontal cortex) (Talavage et al., 2014). In the study by Lipton et al. (2013), cognitive changes were detected above a threshold of 1800 headings. But because of the retrospective nature of the study, in which soccer players had to remember how many headings they had done in the past, the threshold should be considered with caution. In this study, participants made a total number of 400 headings, around 100 headings per session, which is far less than 1800 , mentioned above. However, 400 headings were chosen because this fitted to the median of headings per year, mentioned by Lipton et al. (2013). Besides, the number of 100 headings in one session was cho- sen due to the well-established number of headings in a soccer-training unit, according to the German Soccer Association. However, this benchmark is 10 times higher than the reality of the number of headings that occur in a competitive game (Rodrigues, Lasmar, \& Caramelli, 2016). Therefore, the result that women complained more of headaches needs to be interpreted with caution. On the other hand, one might argue that in reality, there might be more than one training session per week. The question of how many headings have to be performed until adverse effects can be expected in a prospective study is difficult to answer. In this study, we increased the number of headings compared to the two prospective studies (Putukian et al., 2000; Rieder \& Jansen, 2011) published to date. Further investigation about the optimum number of headings, which may involve a greater number of headings, must be done carefully and step-by-step, with careful consideration of the ethical issues.

\section{The Role of Gender Concerning the Impact of Heading}

Beside the replication of the null-findings regarding cognitive performance change after one heading session (Rieder \& Jansen, 2012), we replicated the finding that women suffered headaches more frequently during and after the training. Reasons for this might include a higher headache occurrence outside of the football sessions, less neck muscles, or also a higher lack of heading experience of women compared to men. Symmetrical strength in neck muscles, for example, could prohibit neurological symptoms, which might appear from heading. It has been shown that a stiffening effect of the cervical muscles occurs when a soccer player who is trained in heading, anticipates a heading. Also, the strong neck muscles can dampen the oscillations of extensions and flexions during the heading process (Dezman, Ledet, \& Kerr, 2014). The gender-related results really give a hint that more studies concerning gender differences regarding the impact of heading are warranted, as well as the investigation of the reasons 
for this: Do women suffer from physical symptoms more often than males due to different hormonal status, or due to different neck strength, or does the headings experience play an important role?

\section{Limitations and Future Research}

While this study examines possible effects of heading in soccer players, it also has its limitations. First of all, the number of women and men in the three groups differed and should be equalized in further studies. Second, it is still an exploratory study with only a specific number of cognitive measurements. However, it is only the second study, as far as we know, to attempt to control for confounding variables (like IQ) while using a pretest and post-test design and a control. Third, due to the study of Rieder and Jansen (2011), we only expected physical complaints in the heading group, and the Physical Questionnaire was therefore only administered to the heading group. This is a limitation, which should be ameliorated in further studies. Moreover, the design of this study might be worth expanding in further research by varying the number of heading-training sessions and investigating further variables, like neck strength, hormones, or also the age of first exposure to soccer or football. The latter might influence brain structure before the testing (e.g., Stamm, Bourlas et al., 2015; Stamm, Koerte et al., 2015). Also, to understand the neurological and neuropsychological consequences of heading in more detail, it is important to conduct prospective studies over years with professional as well as amateur football teams.

\section{Conclusion}

To conclude, this prospective study with a pretest and post-test design, and a control group did not show any cognitive impairment immediately after a heading-training of one and four sessions with 400 headings. However, significantly more women than men complained of headaches during and after the training.

\section{Conflict of Interest}

None.

\section{Financial Support}

This research received no specific grant from any funding agency, commercial or not-for-profit sectors.

\section{Ethical Standards}

The authors assert that all procedures contributing to this work comply with the ethical standards of the relevant national and institutional committees on human experimentation and with the Helsinki Declaration of 1975, as revised in 2008.

\section{Acknowledgment}

We thank Simon Glöbl and Ralph Rusu for helping during data acquisition.

\section{References}

Brickenkamp, R. (2002). Test d2 - Aufmerksamkeitsbelastungs-test. Göttingen: Hogrefe.

Colvin, A.C., Mullen, J., Lovell, M.R., West, R.V., Collins, M.W., \& Groh, M. (2009). The role of concussion history and gender recovery from soccerrelated concussion. American Journal of Sports Medicine, 37, 1699-1704.

Covassin, T., \& Elbin, R.J. (2011). The female athlete: The role of gender in the assessment and management of sport-related concussion. Clinical Sports Medicine, 20, 125-131.

Covassin, T., Swanik, C.B., \& Sacks, M.L. (2003). Sex differences and the incidence of concussions among collegiate athletes. Journal of Athletic Training, 38, 238-244.

Dezman, Z.D.W., Ledet, E.H., \& Kerr, H.A. (2014). Neck strength imbalance correlates with increased head acceleration in soccer heading. Sports Health, 5, 320-326.

Dick, R.W. (2009). Is there a gender difference in concussion incidence and outcomes? British Journal of Sports Medicine, 43(Suppl. 1), 46-50.

Dorminy, M., Hoogeveen, A, Tierney, R.T, Higgins, M, McDevitt, J.K, \& Kretzschmar, J. (2015). Effect of soccer heading ball speed on S100B, sideline concussion assessments and head impact kinematics. Brain Injury, 25, 1-7.

Dvorak, J., \& Junge, A. (2000). Football injuries and physical symptoms. The American Journal of Sports Medicine, 28, 3-9.

Elbin, R.J., Beatty, A., Covassion, T., Schatz, P., Hydeman, A., \& Kontos, A.P. (2015). A preliminary examination of neurocognitive performance and symptoms following a bout of soccer heading in athletes wearing protective soccer headbands. Research in Sports Medicine: An International Journal, 23, 203-214.

Gronwall, D. (1977). Paced auditory serial-addition task: A measure of recovery from concussion. Perceptual and Motor Skills, 44, 367-373.

Katis, A., Kellis, E., \& Lees, A. (2015). Age and gender differences in kinematics of powerful instep kicks in soccer. Sports Biomechanis, 3, 287-299.

Koerte, I.K., Ertl-Wagner, B., Reiser, M., Zafonte, R., \& Shenton, M.E. (2012). White matter integrity in 
the brains of professional soccer players without a symptomatic concussion. Journal of the American Medical Association, 308, 1859-1861.

Koerte, I.K., Mayinger, M., Muehlmann, M., Kaufmann, D., Lin, A. P., Steffinger, D., ... \& Heinen, F. R. (2016). Cortical thinning in former professional soccer players. Brain Imaging and Behavior, 1-7 https:// www.ncbi.nlm.nih.gov/pubmed/26286826.

Lephart, S.M., Ferris, C.M., Riemann, B.L., Myers, J.B., \& Fu, F.H. (2002). Gender differences in strength and lower extremity kinematics during landing. Clinical Orthopaedics and Related research, 401, 162-169.

Lipton, M.L., Namhee, K., Zimmerman, M.E., Kim, M., Stewart, W., Branch, C.Q. \& Lipton, R.B. (2013). Soccer heading is associated with white matter microstructural and cognitive abnormalities. Radiology, 268, 850-857.

Maher, M.E., Hutchinson, M., Cusimano, M., Comper, P., \& Schweizer, T.A. (2014). Concussions and heading in soccer: A review of the evidence of incidence, mechanisms, biomarkers and neurocognitive outcome. Brain Injury, 28, 271-285.

McCaffrey, R.J., Westervelt, H.J., \& Haase, R. (2001). Serial neuropsychological assessment with the National Institute of Mental Health (NIMH) Aids Abbreviated Neuropsychological Battery. Archives of Clinical Neuropsychology, 16, 9-18.

Oswald, W.D., \& Roth, E. (1987). Der ZahlenVerbindungs-test ZVT. Göttingen: Hogrefe.

Peters, M., Chisholm, P., \& Laeng, B. (1995). Spatial ability, student gender, and academic performance. Journal of Engineering Education, 84, 60-73.

Putukian, M., Echemendia, R.J., \& Mackin, S. (2000). The acute neuropsychological effects of heading in soccer. A pilot study. Clinical Journal of Sport Medicine, 10, 104-109.

Reitan, R.M. (1956). Trail making test. Manual for administration, scoring, and interpretation. Indianapolis, IN: Indiana University Press.
Rieder, C., \& Jansen, P. (2011). No neuropsychological consequences in male and female soccer players after short heading training. Archives of Clinical Neuropsychology, 26, 583-591.

Rodrigues, A.C., Lasmar, R.P., \& Caramelli, P. (2016). Effects of soccer heading on brain structure and function. Frontiers in Neurology, 7, 38.

Rutherford, A., \& Stephens, R. (2007). Does football heading impair cognitive function? In W.P. Briscoe (Hrsg.), Focus on cognitive disorder research (pp. 181-226). Hauppauge NY: Nova Science Publishers.

Stamm, J.M., Bourlas, A.P., Baugh, C., Fritts, N.G., Daneshvar, D., Martin, B.M., .. . Stern, R.A. (2015). Age of first exposure to football and later-life cognitive impairment in former NFL players. Neurology, 84, 1114-1120.

Stamm, J.M., Koerte, I.K. et al. (2015). Age at first exposure to football is associated with altered corpus callosum white matter microstructure in former professional football players. Journal of Neurotrauma, 15, 1768-1776.

Stephens, R., Rutherford, A., Potter, D., \& Fernie, G. (2010). Neuropsychological consequence of soccer play in adolescent U.K. school team soccer players. Journal of Neuropsychiatry and Clinical Neuroscience, 22, 295-303.

Talavage, T., Nauman, E.A., Breedlove, E.L., York, U., Dye, A., Morigaki, K., ... Leverenz, L.J. (2014). Functionally-detected cognitive impairment in high school football player without clinically-diagnosed concussion. Journal of Neurotrauma, 31, 327-338.

Vandenberg, S.G., \& Kuse, A.R. (1978). Mental rotations, a group test of 3-dimensional spatial visualization. Perceptual and Motor Skills, 47, 599-604.

Witol, A.D., \& Webbe, F.M. (2003). Soccer heading frequency predicts neuropsychological deficits. Archives of Clinical Neuropsychology, 18, $397-417$. 